\title{
A inserção da reabilitação psicossocial nos serviços extra-hospitalares de saúde mental:o conflito entre racionalidade instrumental e racionalidade prática
}

\author{
The inclusion of psychosocial rehabilitation in \\ outpatient mental health services: the conflict \\ between instrumental rationality and practical \\ rationality
}

\author{
Regina Célia Fiorati ${ }^{1}$, Toyoko Saeki
}

\begin{abstract}
FIORATI, R. C.; SAEKI, T. A inserção da reabilitação psicossocial nos serviços extra-hospitalares de saúde mental:o conflito entre racionalidade instrumental e racionalidade prática. Rev. Ter. Ocup. Univ. São Paulo, v. 22, n. 1, p. 76-84, jan./abr. 2011.

RESUMO: A partir de uma pesquisa desenvolvida no município de Ribeirão Preto, SP, realizada em dois serviços extra-hospitalares de saúde mental, um Ambulatório Regional de Saúde Mental e um Centro de Atenção Psicossocial II, objetivou-se estudar a organização dos serviços extrahospitalares, os projetos terapêuticos desses serviços e a inserção da reabilitação psicossocial nos projetos terapêuticos. A pesquisa foi realizada com base em metodologia qualitativa, como técnicas de coleta de dados utilizou-se entrevistas semidiretivas e grupos focais, e a análise dos dados foi realizada com base em referencial hermenêutico dialético de Jürgen Habermas, seguindo uma técnica interpretativa reconstrutiva. Os resultados foram a identificação de que o trabalho nesses serviços se organiza com centralidade no trabalho clínico-psiquiátrico, enfatiza o diagnóstico e a patologia, o tratamento centrado no medicamento e a reabilitação psicossocial é secundarizada nos projetos e ações disponibilizadas aos usuários. $\mathrm{O}$ artigo traz discussão sobre parte desse estudo no que diz respeito à inserção da reabilitação psicossocial nos serviços estudados, enfocando o assunto sob o prisma do conflito entre dois tipos de racionalidade, a instrumental e a prática.
\end{abstract}

DESCRITORES: Terapia ocupacional; Reabilitação; Centros de reabilitação; Serviços de saúde mental; Psiquiatria.

\footnotetext{
* Este artigo é parte da Tese de doutorado intitulada "A organização dos serviços extra-hospitalares de saúde mental, o projeto terapêutico e a inserção da reabilitação psicossocial” apresentada à Escola de Enfermagem de Ribeirão Preto da Universidade de São Paulo, cuja defesa pública e aprovação da mesma se deu em 04/10/2010.

1. Terapeuta Ocupacional, doutora em Ciências pelo Programa de Pós-Graduação em Enfermagem Psiquiátrica da Escola de Enfermagem de Ribeirão Preto, Universidade de São Paulo. Docente do Curso de Terapia Ocupacional do Departamento de Ciências Biológicas e da Saúde do Centro Universitário de Araraquara.

2. Enfermeira. Docente da Escola de Enfermagem de Ribeirão Preto, Universidade de São Paulo.

Endereço para correspondência: Regina Célia Fiorati. R. Graciliano Ramos, 100, AP.21. Jardim Antártica, Ribeirão Preto - CEP. 14051039. e-mail: reginafiorati@yahoo.com.br
} 


\section{INTRODUÇÃO}

$\mathrm{O}$ s serviços extra-hospitalares de saúde mental foram criados com base nas iniciativas a partir das reformulações da atenção psiquiátrica advindas dos Movimentos de Reforma Psiquiátrica. A Lei que regulamenta a reestruturação psiquiátrica no Brasil dá ênfase na substituição progressiva da atenção manicomial por novos dispositivos de tratamento e acolhimento na comunidade, fora das estruturas hospitalares. Nesse sentido, esses serviços desde sua criação, têm a missão ético-política de substituição de um modelo de atenção psiquiátrica centrado no hospital e nas formas de exclusão do portador de sofrimento psíquico, por um modo de atenção psicossocial, o qual tem a função de fornecer tratamento ampliado aos usuários, no que diz respeito às ações clínicas e de reabilitação, tendo como um de seus objetivos centrais a inclusão social do portador de sofrimento mental (BRASIL, 2004).

Por meio de uma pesquisa desenvolvida em dois serviços extra-hospitalares de saúde mental no município de Ribeirão Preto, Estado de São Paulo, observou-se que alguns problemas, apresentados na base da constituição e funcionamento desses serviços, colocavam-se como entraves importantes na consolidação de um modo efetivo de organização psicossocial na atenção disponibilizada aos usuários, mantendo-se, ainda, nesses serviços, características de um modelo de tratamento centrado no diagnóstico psiquiátrico, na psicopatologia e no trabalho do profissional médico. De tal forma que as ações de reabilitação psicossocial se encontravam em posição periférica, ou mesmo inexistentes nos serviços estudados.

A intenção, neste trabalho, é apresentar a discussão sobre parte dessa pesquisa no que se refere à inserção da reabilitação psicossocial nos projetos terapêuticos dos serviços pesquisados. Além disso, trata-se de interpretar as concepções que os profissionais dos serviços elaboram sobre a reabilitação psicossocial e enfocar o problema do ponto de vista que explora uma visão na qual a racionalidade instrumental e científica que opera a ênfase medicocentrada do tratamento disponibilizado, impõe-se a uma racionalidade prática que se aplica as ações de reabilitação psicossocial.

A seguir, serão apresentadas as informações sobre a pesquisa desenvolvida e a metodologia empregada e após passa-se a discussão do problema que se quer enfocar neste trabalho.

\section{A pesquisa e percurso metodológico: um enfoque interpretativo e participativo}

A pesquisa foi desenvolvida no município de Ribeirão Preto, interior do Estado de São Paulo, no período de 2007 a 2009, e em dois serviços extra-hospitalares de saúde mental: um Ambulatório Regional de Saúde Mental e um CAPS II. O primeiro se caracteriza por ser o maior serviço extra-hospitalar de saúde mental do município e atender um maior número de usuários e o segundo por se constituir no único nessa modalidade em um município de aproximadamente 560.000 habitantes (RIBEIR ̃̃O PRETO, 2009).

Os objetivos da pesquisa foram analisar a organização dos serviços selecionados para o estudo, o projeto terapêutico e a inserção da reabilitação psicossocial nos projetos terapêuticos.

Os sujeitos da pesquisa foram os profissionais de nível superior e médio ligados diretamente a assistência ao usuário dos serviços selecionados.

A metodologia desenvolvida se deu com base na abordagem qualitativa e as técnicas de coleta de dados foram entrevistas semidiretivas e grupos focais. As entrevistas tinham um eixo central que solicitava ao participante que descrevesse seu trabalho no serviço, mencionasse como era elaborado o projeto terapêutico pela equipe e referisse sua concepção de reabilitação psicossocial. Em um segundo momento da pesquisa, após uma primeira análise das entrevistas, alguns eixos temáticos, os quais se referiam à predominância dos procedimentos de caráter puramente clínico e a subvalorização da reabilitação psicossocial nas ações terapêuticas, foram submetidos aos profissionais dos serviços estudados, por meio de grupos focais, para que discutissem os eixos assinalados e, dessa forma, o objetivo era garantir que a pesquisa se desse por meio de uma metodologia participativa e com a construção compartilhada do conhecimento (ONOCKO-CAMPOS; FURTADO, 2001).

Foram realizadas dezenove entrevistas com os seguintes profissionais: quatro auxiliares de enfermagem, duas enfermeiras, duas psicólogas, duas terapeutas ocupacionais, três assistentes sociais, um auxiliar de serviço de saúde, três psiquiatras e dois gerentes.

Nos grupos focais participaram dezenove profissionais, dentre esses: dois auxiliares de enfermagem, três enfermeiras, quatro psiquiatras, três psicólogas, duas terapeutas ocupacionais, três assistentes sociais e dois gerentes. Os sujeitos que participaram dos grupos focais, em sua maioria, foram os mesmos que participaram das entrevistas, porém, alguns não se encontravam nos serviços no dia da realização dos encontros grupais, sendo que outros foram incluídos. Nos grupos focais foi apresentada uma análise sumária das entrevistas e um eixo para a 
discussão que indagava por que nos serviços o tratamento sobrepunha a ação médico-psiquiátrica às de reabilitação psicossocial?

Oreferencial teórico que orientou metodologicamente a pesquisa foi a filosofia hermenêutica dialética de Jürgen Habermas que propiciou uma análise dos dados apoiada na interpretação e compreensão das construções discursivas e concepções dos autores-sujeitos da pesquisa ao invés de uma análise apoiada na descrição de fatos e explicação de fenômenos (HABERMAS, 1987, 1988).

Os procedimentos éticos observados na pesquisa foram a submissão do projeto ao Secretário Municipal de Saúde do Município de Ribeirão Preto e ao Comitê de Ética em Pesquisa da Escola de Enfermagem de Ribeirão Preto, da Universidade de São Paulo, com a aprovação do mesmo em outubro de 2007, sob protocolo $\mathrm{n}^{\circ} 0831 / 2007$ . No início de cada entrevista foi entregue um Termo de Consentimento Livre e Esclarecido, explicado o seu conteúdo e assinado pelos dois participantes, pesquisador e sujeito. Foi entregue outro Termo de Consentimento Livre e Esclarecido para os integrantes dos grupos focais e foram seguidos os mesmos procedimentos anteriores.

A seguir são apresentados os dados referentes à discussão enfocada neste artigo: a inserção da reabilitação psicossocial nos projetos terapêuticos e ações disponibilizadas nos serviços extra-hospitalares de saúde mental selecionados no estudo referido. No próximo tópico discutem-se as concepções que os profissionais desses serviços elaboram sobre a reabilitação psicossocial.

\section{As concepções sobre a reabilitação psicossocial: cisão entre clínica e reabilitação}

As dificuldades psicossociais apresentadas pelas pessoas com transtornos mentais e a necessidade da criação de ações, nos serviços, que respondam a essas dificuldades, são reconhecidas em todos os discursos contidos nas entrevistas. Os profissionais reconhecem que as questões fundamentais do processo da reforma psiquiátrica encontram-se ainda em caminho de construção e enfrentamento, e que a continuidade dos processos de mudanças, incluindo suas práticas profissionais, tem que vislumbrar ações concretas para aproximar as experiências existenciais da loucura dos espaços físicos, socioculturais e de legitimidade cidadã.

As ações de reabilitação psicossocial não são desenvolvidas nos serviços pesquisados, embora são consideradas como necessárias, porém de difícil realização concreta, devido a variados fatores que vão desde a carência de recursos materiais e humanos, falta de tempo disponível dos profissionais. Em outros casos, são concebidas como atividades que não fazem parte do campo das responsabilidades dos serviços e que, portanto, deveriam ser oferecidas em outros locais.

Mas se nos discursos a reabilitação psicossocial é mencionada como dispositivo necessário, por que na prática ela não é implementada ou efetivada com consistência pelos profissionais, nem contemplada com excelência pelos projetos terapêuticos?

Para aproximarmo-nos desse aparente paradoxo é necessário analisarmos as concepções que os profissionais dos serviços do estudo mantêm sobre a reabilitação psicossocial.

Três concepções básicas podem ser destacadas da análise das entrevistas, sendo que: a primeira, elaborada pelos profissionais que fizeram ou fazem parte de grupos ligados a movimentos de luta antimanicomial e lutaram diretamente, ou lutam ainda, pelos objetivos da reforma psiquiátrica, está imbuída nos propósitos do modelo psicossocial. A segunda concepção encontrada nos relatos dos profissionais associa a reabilitação psicossocial a alguma forma de normalização das condutas e habilidades dos sujeitos tratados, segundo uma esfera pedagógica; identificam-na com a terapia ocupacional, ou associamna à capacidade dos usuários aprenderem e reproduzirem condutas viáveis na sociedade, bem como, para adquirirem alguma habilidade ocupacional. O terceiro recorte que efetuamos na análise diz respeito à concepção de reabilitação psicossocial que se assemelha ao segundo recorte, porém remete a reabilitação a um lugar idealizado, adequado para realizá-la. O serviço é o local para o tratamento, a reabilitação seria atividade própria para as oficinas abrigadas, centros de convivência, ou outros locais que não o espaço do serviço.

Vejamos mais detalhadamente cada uma delas.

A primeira concepção diz respeito aos profissionais que estiveram engajados nos movimentos de reformulação da atenção psiquiátrica nas décadas de 1980 e 1990. Esses movimentos foram os responsáveis pelas mudanças efetuadas no modelo de tratamento e abordagem ao sofrimento psíquico que propôs a substituição dos estabelecimentos asilares por serviços abertos e comunitários com base extra-hospitalar.

Além disso, as transformações realizadas, e ainda em vias de construção, ocorreram em diversas áreas da esfera das ações sociais, não só do ponto de vista do campo técnico-assistencial, mas também do entendimento teóricoconceitual do modelo antigo da psiquiatria, propondo sua desconstrução e a reconstrução de uma epistemologia que focasse, em primeiro lugar, o sujeito em sofrimento 
e não a doença. Assim como, no campo jurídico-político criaram-se ações no sentido de garantia ao exercício da cidadania dos sujeitos, no campo sócio-cultural pretendeuse transformar as noções presentes no imaginário social no que diz respeito à loucura buscando novas formas de inclusão e lidar com a diferença (AMARANTE, 2007).

Para atingir esses amplos objetivos foi proposta a reorientação das ações nos serviços de saúde mental de modo a propiciar formas de cuidado e atenção que não ficassem restritas, exclusivamente, à atuação clínica, isto é, a atuação de caráter diagnóstica e curativa. Ou seja, além do tratamento clínico, tratamento psiquiátrico-medicamentoso e outras ações terapêuticas que se assemelham à tecnologia médica, a nova orientação propunha a construção de espaços de sociabilidade, de trocas e agenciamento de subjetividades. A proposta pretendeu contemplar um modelo de atendimento ao sujeito com transtornos mentais que superasse a metodologia do modelo hospitalocêntrico, vislumbrando apenas a doença, sendo que a subjetividade da pessoa só era vista sob a ótica da patologia (AMARANTE, 1996).

De acordo com Costa-Rosa (2000), esse processo de mudança pode ser qualificado como uma genuína mudança de paradigma, o da atenção psicossocial.

Sendo assim, alguns agentes que participaram dos movimentos de reformulação da atenção psiquiátrica, após a luta pela desconstrução dos aparatos manicomiais, concentraram seus esforços em criar serviços com projetos terapêuticos ancorados na idéia de reabilitação psicossocial.

Dessa forma, as estratégias da reabilitação psicossocial centraram sua ação na afirmação da autonomia e cidadania do sujeito em sofrimento psíquico, no deslocamento do modelo de atenção biomédico e hospitalar para dispositivos extra-hospitalares, ações territoriais de inclusão, de reconstrução de um cotidiano com sentido existencial pleno e a produção de projetos terapêuticos interdisciplinares que cuidassem dessa pessoa em sofrimento de forma ampliada e integral (SARACENO, 1999).

Os profissionais que contribuem para a construção dessa perspectiva, contudo, não constituem a maioria dos trabalhadores dos serviços. Há, ainda, duas concepções predominantes nos serviços que atrelam a reabilitação psicossocial à concepção de normalização do sujeito doente no sentido de aproximá-lo de padrões idealizados de conduta, comportamentos e funcionamento social. O que difere essas duas últimas concepções é que enquanto a segunda concebe a reabilitação associada à função do profissional terapeuta ocupacional, a terceira não concebe a reabilitação psicossocial como função do serviço de saúde mental no qual trabalham, mas de local idealizado para tanto: complexos de oficinas abrigadas, centros de convivência, entre outros.

Essas duas últimas concepções correspondem a uma visão mais tradicional de reabilitação, conceito que traz embutidos elementos de uma concepção que fundamentou a criação das primeiras ações de reabilitação.

As primeiras noções de reabilitação fundam-se nas premissas de retomada de uma normalidade produtiva ou de funcionamento social adaptado, que habilitaria ou reabilitaria o sujeito desabilitado a viver em sociedade e retomar alguma atividade produtiva no sentido de manter-se em condição ocupacional adequada, mesmo sendo ocupação destituída de sentido criativo e existencial para o sujeito. $\mathrm{O}$ trabalho, nesse caso, não era entendido enquanto um direito social do cidadão, mas enquanto um meio/fim terapêutico que viabilizaria a adaptação do sujeito (desaptado) ao seu meio social e aos ideais dessa sociedade de funcionalidade adaptativa e produtiva (SOARES, 2007).

O terceiro recorte que efetuamos reflete uma concepção dos profissionais dos serviços em estudo, segundo a qual ações de reabilitação psicossocial não são vistas como pertinentes ao serviço ao qual pertencem. Nessa concepção, o serviço constitui-se em espaço específico para tratamento e a reabilitação não é pensada como parte das suas funções, sendo remetida sua viabilidade para um lugar imaginário. Essa visão apresenta um ponto importante para a nossa análise, no que diz respeito à cisão explícita entre tratamentos e reabilitação, entre ação clínica e ação psicossocial e o remetimento da reabilitação como função para ser desenvolvida em espaços apropriados para isso, diferentes dos espaços clínicos

Por que a tarefa da reabilitação psicossocial é remetida para outros serviços, ou espaço imaginário apropriado para a realização da inclusão social? A inclusão social precisa de um espaço e pessoas específicas para isso? Por que a reabilitação e a inclusão social não podem ser desenvolvidas pela mesma equipe que trata?

Para respondermos a essas questões é necessário olharmos mais de perto o porquê dessas duas ações de trabalho próprias aos serviços extra-hospitalares de saúde mental terem sido concebidas como apartadas uma da outra, sendo que a atividade clínica é sobreposta à reabilitação. Veremos, portanto, que há uma tensão entre duas racionalidades correspondentes aos modos conceptuais relacionados às atividades clínicas e à reabilitação psicossocial. 


\section{Racionalidade instrumental e clínica versus racionalidade prática e reabilitação psicossocial}

A reabilitação psicossocial se caracteriza por ser um conjunto de estratégias apoiado em uma postura ética frente ao usuário de resgatar-lhe espaços, expressão e legitimidade. Isso não se realiza em consultórios. Pode até começar nos consultórios, mas tem necessariamente que se encaminhar para o território. Dessa forma, as ações que constituem a atenção psicossocial superam a compreensão de uma clínica desenvolvida em espaços fixos e inauguram formas diferenciadas de atuação terapêutica, e como lembra Saraceno (1996) não dispõe de teorias acabadas como as teorias de ordem científica como as das ciências médicas e das áreas que nasceram sob sua influência.

Reabilitar psicossocialmente em saúde mental é um processo dinâmico que partindo do fio condutor da história particular construída na relação vincular terapêutica entre terapeuta e usuário prossegue e amplia-se para a construção de uma concreta qualidade de vida cotidiana que implica, necessariamente, em atender-se as condições de vida que envolve trabalho, organização coletiva, redes de relacionamento interpessoais, as ressiginificações dos projetos de vida, entre outros (CASTRO et al., 2001).

Também envolve o trabalho na dimensão pragmática do cotidiano. Entre as múltiplas realidades que condicionam o universo humano há uma que ocupa uma posição privilegiada por ser a dimensão da realidade que se impõe à consciência de maneira maciça, urgente e intensa, autoriza-lhe a designação de realidade predominante, é a realidade cotidiana. O cotidiano é o palco no qual se desenvolvem as lutas diárias pela sobrevivência, que dá sentido à produção instrumental e de objetos necessários à reprodução da vida humana, bem como se apresenta enquanto mundo intersubjetivo, que se compartilha indefinida e necessariamente com outros homens por meio das contínuas ações de construção do mundo (BERGER; LUCKMANN, 1978).

As práticas de saúde e saúde mental atuais exigem uma intervenção do técnico no sentido de apresentar um compromisso com o potencial de renovação da concepção de saúde, que, ao ser destituída das velhas concepções prometéicas do curativismo, passa a entender o sistema como organismo vivo, incompleto, indeterminado, dinâmico e que a saúde longe de ser concebida como estado de reparação de danos ou genérico bem estar passa a ser vista como produção de vida constante, processo coletivo e cotidiano de produção da vida das populações e dos sujeitos, o que implica em toda a multiplicidade de possibilidades de concepções, ações e criações e sugere, por sua vez, que fazer saúde tem como base a realidade do homem como ser social e ao seu entorno material e simbólico (MANGIA; NICÁCIO, 2001).

O cotidiano, portanto, apresenta-se como terreno privilegiado para a reabilitação psicossocial.

Quando Delgado apud Saraceno (1999), refere-se à "formidável banalidade da vida quotidiana", é a essa dimensão que quer salientar e que constitui o terreno fundamental do exercício da cidadania para os sujeitos sociais. A condição humana de poder habitar um espaço mundano, trocar bens materiais e simbólicos objetiva e intersubjetivamente e relacionar-se em rede familiar e social.

Em outra perspectiva, as ações de caráter clínico e o seu dispositivo central, o medicamento, são ações reservadas a especialistas, encerram-se em uma prática sistematizada pelo conhecimento técnico-científico e que são desenvolvidas em espaços determinados especificamente para serem desenvolvidos e aplicados à clientela (FIORATI, 2010).

Ao mesmo tempo, vemos que a terapêutica médico-psiquiátrica assume maior relevância em relação às ações de saúde como um todo e observamos que nos serviços extra-hospitalares de saúde mental que estudamos, o medicamento torna-se o dispositivo absoluto de credibilidade curativa, adquirindo um valor apodítico frente a outras atuações terapêuticas, ao ser considerado como o Bem Supremo, intervenção indispensável, portador de uma promessa de eficiência máxima diante do sofrimento.

É evidente que há interesses claros da organização econômico-política na reprodução da idéia de uma dimensão mágica de curabilidade transposta ao dispositivo medicamentoso e sua propagação como mercadoria de consumo de primeira linha, que são as indústrias farmacêuticas e os agentes que ganham economicamente com o mercado farmacêutico (AMARANTE, 1996).

Entretanto, o privilégio e a supervalorização da atividade clínico-medicamentosa acompanhados da subvalorização de atividades encampadas pela reabilitação psicossocial, não se explicam apenas do ponto de vista econômico e político ao favorecer determinados segmentos sociais que lucram com o mercado de psicofármacos e pela ideologia cientificista que paramenta a área médica e suas subáreas de maior cientificidade, retirando o mesmo das ações psicossociais. Há também uma compreensão desse fato de uma perspectiva da racionalidade que acompanha a supervalorização técnica em nossa sociedade e desvaloriza uma racionalidade prática.

As ações de saúde, segundo Ayres (2008), contam 
com um poderoso arsenal tecnocientífico voltado para seu êxito instrumental e uma frágil base conceitual para compreensão dos processos de saúde com base em uma racionalidade prática voltada para o sentido do cuidado em saúde.

De acordo com o autor, há três diferentes esferas da racionalidade presentes nas áreas da experiência humana. A primeira esfera diz respeito aos conhecimentos universais, das verdades perenes e das causas, que fundamenta a racionalidade cognitiva da epistemologia da ciência moderna. A segunda esfera diz respeito à atividade de produção de artefatos, da criação de objetos que, por meio da razão, acumula um saber fazer voltado para a produção de bens e instrumentos para vida humana, que constitui o conjunto de saberes, base de uma racionalidade instrumental. E a terceira, a racionalidade prática, é constituída pela ação humana que visa à sabedoria prática, não está voltada para a universalidade, alimenta-se da contingência, volta-se para a produção de conhecimentos que visam à estruturação da convivência humana satisfatória no que diz respeito a garantir-se a Vida Boa e movida pela construção de valores que se dirigem sempre para o Bem Comum. Essa é a esfera racional da praxis. Este é o campo que marca a ação política dos homens.

Utilizando essa reflexão do autor referido podemos pensar a relação entre as práticas médico-clínicas e a reabilitação psicossocial, dizendo que as práticas médicas se fundamentam nas duas primeiras esferas da racionalidade, a cognitiva e a técnica ou instrumental, ou seja, buscam seus fundamentos na racionalidade científica das ciências biológicas e constroem determinadas técnicas e instrumentos cuja finalidade é o êxito técnico da ação curativa.

Dessa maneira, as práticas médicas que são fundamentadas nas teorias cientificas e técnicas correspondentes, caminham no terreno das verdades muito bem estabelecidas com base na construção sistemática do conhecimento, detentoras da força de uma legitimidade forjada em instituições cujo poder tem a condição da expressão cultural dominante em nossa sociedade.

Ao contrário, a reabilitação psicossocial constrói-se, eminentemente, segundo uma racionalidade prática. Isto é, as ações psicossociais se dão no terreno dos contextos das necessidades de saúde dos usuários, expressas por meio de aspectos diferenciados de acordo com os distintos contextos culturais, sociais, econômicos e políticos. Tratase de ações que buscam a democratização das condições da Vida Boa para pessoas em estado de exclusão desses espaços de produção social da vida, a inclusão dessas pessoas nos espaços e instâncias da vida coletiva que discute e constrói, permanentemente, o Bem Comиm. Em outras palavras, estamos no terreno franco da práxis e, portanto, da dimensão política da existência humana.

Dessa forma, vemos que há uma cisão entre as duas práticas, sustentada por uma tensão entre duas formas de racionalidade que comandam as ações de saúde nos serviços.

Por um lado, estão as práticas clínico-médicas estabelecidas pela tradição e pelo peso da respeitabilidade das instituições e produções científicas, a operação ideológica que garante a primazia dessas práticas sobre outras, sua capacidade de concentrar um valor simbólico, reproduzido pela mídia e sistemas de comunicação, como detentoras de eficiência máxima sobre os processos de adoecimento e dor, e representantes de um conhecimento considerado total, absoluto e apodítico sobre as doenças, os doentes e a cura. Por outro lado, estão as práticas da reabilitação psicossocial que, segundo uma racionalidade prática, buscam a inclusão dos portadores de sofrimento psíquico nas redes sociais de produção e reprodução da vida no mundo, mas que, por sua vez, não detêm um arsenal tecnocientífico para se legitimarem, não trabalham na determinação de conhecimentos universais e sim dentro dos contextos cambiantes da tessitura sociocultural, com conceitos provisórios destinados a responderem a problemáticas pontuais e contingenciais da vida cotidiana dos usuários e de seus familiares.

Os serviços de saúde e saúde mental, entretanto, necessitam reunir essas duas dimensões terapêuticas no conjunto das práticas de cuidado que disponibilizam.

De acordo com Ayres (2004), as práticas médicas centralizadas apenas na dimensão instrumental tecnocientífica têm sido amplamente aplicadas nos serviços de saúde sem, contudo, obterem a eficácia máxima que prometem, permanecendo insatisfações com o sistema. Portanto, a instrumentalidade técnica como forma centralizadora das ações de saúde, apesar de não ser, de forma geral tão eficiente quanto promete, continua, paradoxalmente, sendo reproduzida de forma maciça nos serviços.

Para o autor, é necessário ultrapassar a aplicação dos saberes técnicos indispensáveis, mas, por certo, também é imperativo pensar em uma nova clínica que se apóie no encontro humano entre os sujeitos envolvidos e que junto com a questão técnica medicamentosa e curativa siga-se uma preocupação por parte do terapeuta de se evidenciar as condições subjetivas e simbólicas que envolvem o sujeito e sua experiência de adoecer, sendo imprescindível, para o terapeuta, a utilização de conhecimentos com base em uma racionalidade prática, que marcaria mais a dimensão do cuidado. 
Sendo assim, colocamo-nos a mesma questão: como garantir a reunião, nos projetos terapêuticos dos serviços extra-hospitalares de saúde mental, dessas duas esferas das ações terapêuticas: a ação clínica e a reabilitação psicossocial?

\section{Tratamentos e reabilitação: uma relação} possível?

À questão colocada de como reunir essas duas ações de saúde nos serviços extra-hospitalares de saúde mental, antecedemos outra: essa reunião é possível? Acreditamos que sim. Para tanto, é necessária uma transformação na concepção de clínica e uma reorientação das concepções de reabilitação psicossocial, consolidando concepções, realmente, ligadas ao paradigma do modo de atenção psicossocial.

Um usuário, frequentemente, necessita de ajuda para realizar projetos de vida com determinadas qualidades que revalorize as experiências vitais cotidianas, assim como, necessita de ajuda para a realização de ações que o recoloquem em contato com a vida, social, cultural e política.

Quem é o agente responsável por tais ações? Refaçamos a pergunta: por que o mesmo agente que clinica não pode se responsabilizar por tais iniciativas no campo psicossocial?

Para tanto, é necessária uma ampliação na concepção de clínica. É necessário que se crie uma clínica que, segundo as proposições de Ayres (2004, 2007), englobe tanto ações tecnocientíficas que visem êxito perante os processos patológicos, bem como ações que visem um sucesso prático para o usuário para que se promova, realmente, vida com qualidade.

A reabilitação psicossocial, por sua vez, não detém um rol de procedimentos técnicos, nem se apóia em sistemas acabados de conhecimento. Não se localiza dentro da dimensão das tecnociências, nem visa, em primeiro plano, um êxito técnico, mas um êxito no campo pragmático da vida do usuário de forma a qualificar sua existência, ou seja, visa, em primeiro plano, um sucesso prático.

Dessa forma, a reabilitação psicossocial deixa de ser entendida enquanto procedimento, para ser compreendida como conceito segundo o qual o ato de reabilitar não se distingue do ato de tratar, mas, justamente, o ato de tratar envolve necessariamente a dimensão da busca prática por demandas que ultrapassem a mera remissão dos sintomas para entender o processo de saúde do sujeito como um percurso que inclui a reflexão e construção de um projeto de vida global, que seja significativo e que lhe forneça meios para a apropriação de autonomia, de valor social e de emancipação.

\section{CONSIDERAÇÕES FINAIS}

As orientações da Reforma Psiquiátrica e as Políticas Públicas no campo da Saúde Mental que as incluíram em grande escala, são claras em ressaltar a importância e a relevância que deve ser dada, nos projetos terapêuticos dos serviços substitutivos e extra-hospitalares de saúde mental, às ações de inclusão do portador de sofrimento psíquico na comunidade. Vemos, entretanto, que nos serviços estudados a ênfase da atenção apresentase sobre a patologia, o diagnóstico, no tratamento clínico-medicamentoso com enfoque predominante sobre a remissão de sintomas, segundo uma abordagem curativista.

Evidenciamos que há nos serviços pesquisados uma organização do trabalho centrado na ação médico-clínica, e observamos que ações de reabilitação psicossocial são praticamente ausentes nos serviços. As ações de saúde voltam-se muito para a inclusão do usuário no próprio serviço, mas não na sociedade.

Os profissionais apresentam concepções obsoletas sobre a reabilitação tais como formas de busca de normalidades produtivas, de aquisição de habilidades sociais para condutas uniformes e hegemônicas e encaram tal função como totalmente apartada do exercício da clínica.

Dessa forma, a reabilitação psicossocial nos serviços estudados é quase inexistente sendo sempre remetida, de forma idealizada, a sua realização a outros espaços que não o do serviço ou a outros profissionais que teriam como especialidade o seu desenvolvimento.

Apontamos a necessidade premente de reunir nas esferas dos projetos e ações de saúde dos serviços os tratamentos clínicos e a reabilitação psicossocial, mesmo que tenhamos que promover uma transformação nas concepções e práticas clínicas e nas concepções e práticas de reabilitação psicossocial. É necessária a criação de fóruns dialógicamente constituídos para que as equipes de profissionais possam rediscutir questões importantes a respeito da forma de organização do trabalho nos serviços extra-hospitalares, seu funcionamento e as ações que são disponibilizadas aos usuários e que possam, sobretudo, produzir coletivamente um projeto terapêutico ampliado e verdadeiramente inserido em paradigma psicossocial. 
FIORATI, R. C.; SAEKI, T. The inclusion of psychosocial rehabilitation in outpatient mental health services: the conflict between instrumental rationality and practical rationality. Rev. Ter. Ocup. Univ. São Paulo, v. 22, n. 1, p. 76-84, jan./abr. 2011.

\begin{abstract}
This study was developed in Ribeirão Preto, SP, Brazil in two outpatient mental health services: a Regional Outpatient Mental Health service and a Psychosocial Care Center II and aimed to investigate the organization of the outpatient care services, the therapeutic projects of these services, and the inclusion of psychosocial rehabilitation in therapeutic projects. It was based on qualitative methodology and used semi-structured interviews and focus groups. Data analysis was based on the hermeneutic dialectic framework of Jürgen Habermas, according to a reconstructive interpretative technique. The results indicated that the organization of these services is focused on the clinical-psychiatric practice, emphasizes the diagnosis and pathology, treatment is medication-centered, and psychosocial rehabilitation is a secondary element addressed in projects and actions available to the services' users. This article discusses part of this larger study in relation to the inclusion of psychosocial rehabilitation in the studied services, addressing the subject in the light of the conflict between two types of rationality: instrumental and practical.
\end{abstract}

KEY WORDS: Occupational therapy; Rehabilitation; Rehabilitation centers; Mental health services; Psychiatry.

\title{
REFERÊNCIAS
}

AMARANTE, P. O homem e a serpente: outras histórias para a loucura e a psiquiatria. Rio de Janeiro: Fiocruz, 1996.

AMARANTE, P. Saúde mental e atenção psicossocial. Rio de Janeiro: Fiocruz, 2007.

AYRES, J. R. C. M. O cuidado, os modos de ser (do) humano e as práticas de saúde. Saúde e Sociedade, São Paulo, v. 13 n. 3, p. 16-29, 2004.

AMARANTE, P. Uma concepção hermenêutica da saúde. Physis: Revista Saúde Coletiva, Rio de Janeiro, v. 17 n. 1, p. 43-62, 2007.

AMARANTE, P. Para comprender el sentido practico de lãs aciones de salut: contribuiciones de la hermenêutica filosófica. Salut Coletiva, Universidad Nacional de Lanús, Argentina, v. 4, p. 159-172, 2008.

BERGER, P.; LUCKMANN, T. A construção social da realidade: tratado de sociologia do conhecimento. Petrópolis: Vozes, 1978.

BRASIL, Ministério da Saúde. Secretaria-Executiva. Secretaria de Atenção à Saúde. Legislação em saúde mental: 1990-2004. 5a. ed. Brasília, 2004.

CASTRO, E. D.; LIMA, E. M. F. A.; BRUNELlO, M. I. B. Atividades humanas e terapia ocupacional. In: DE CARLO, M. M. P.; BARTALOTTI, C. C. (Orgs). Terapia ocupacional no Brasil: fundamentos e perspectivas. São Paulo: Plexus, 2001, cap. 2, p.41-59.

COSTA-ROSA, A. O modo psicossocial: um paradigma das práticas substitutivas ao modo asilar. In: AMARANTE, P. (Org). Ensaios, subjetividade, saúde mental, sociedade. Rio de Janeiro: Fiocruz, 2000. cap. 8, p. 141-168.

FIORATI, R. C. A organização dos serviços extra-hospitalares de saúde mental, o projeto terapêutico e a inserção da reabilitação psicossocial. 2010. 242f. Tese de doutorado. Programa de PósGraduação em Enfermagem Psiquiátrica. Escola de Enfermagem de Ribeirão Preto. Universidade de São Paulo, Ribeirão Preto, SP.

HABERMAS, J. Técnica e ciência como ideologia. Lisboa: Edições 70, 1987.

HABERMAS, J. Teoría de La acción comunicativa. Madrid:Taurus, 1988.

MANGIA, E. F.; NICÁCIO, F. Terapia ocupacional em saúde mental: tendências principais e desafios contemporâneos. In: CARLO, M.; BERTALOTTI,C. (Orgs). Terapia ocupacional no Brasil. São Paulo: Plexus Editora, 2001. cap. 3. p. 63-80.

ONOCK-CAMPOS, R.; FURTADO, J. P. Entre a saúde coletiva e a saúde mental: um instrumental metodológico para avaliação da rede de Centros de Atenção Psicossocial (CAPS) do Sistema Único de Saúde. Caderno de Saúde Pública, Rio de Janeiro, v. 25, n. 58, p. 98-111, 2001.

RIBEIRÃO PRETO. Secretaria Municipal de Saúde. Plano de Saúde de Ribeirão Preto-2010-2013. Ribeirão Preto, 2009. Disponível em: <www.ribeiraopreto.sp.gov.br/ssaude/16principal. php>. Acesso em: 02 abr. 2010. 
FIORATI, R. C.; SAEKI, T. A inserção da reabilitação. Rev. Ter. Ocup. Univ. São Paulo, v. 22, n. 1, p. 76-84, jan./abr. 2011.

SARACENO, B. Reabilitação psicossocial: uma estratégia para a passagem do milênio. In: PITTA, A. (Org). Reabilitação psicossocial no Brasil. São Paulo: Hucitec, 1996. cap.1. p. 13-18.

SARACENO, B. Libertando identidades. Belo Horizonte: Tè

Recebido para publicação: 24/10/10

Aceito para publicação: 11/04/11
Corá?Instituto Franco Baságlia, 1999.

SOARES, L. B. T. A história da terapia ocupacional. In: CAVALCANTI, A.; GALVÃO, C. (Orgs). Terapia ocupacional: fundamentação \& prática. Rio de Janeiro: Guanabara, 2007. cap 1. p. 3-9. 